\title{
Enhanced New Clustering Ant Colony Optimization based Routing Protocol AODV-R
}

\author{
Harmanjot Singh \\ Guru Nanak Dev University \\ Amritsar, Punjab
}

\author{
Pahulpreet Singh \\ Guru Nanak Dev University \\ Amritsar, Punjab
}

\begin{abstract}
Vehicular Ad hoc Networks (VANETS) tend to be specific type of wireless network created by vehicles interacting between themselves as well as along with roadside device stations. A number of products and services have been produced with regards to VANETS varying from security to comfort applications. An essential need for this kind of services is because they are available with Quality of Service (QoS) ensures with the regard of service consistency as well as availability. This paper represents about the AODV-R routing protocol outperforms in terms of reliability. This paper proposes a New clustering Ant Colony Optimization based routing protocol AODV-R for removing the congestion as well as finding shortest path selection. The proposed methodology has shown quite significant improvement over available ones.
\end{abstract}

\section{Keywords}

VANETS, AODV-R, Clustering Ant colony optimization

\section{INTRODUCTION}

Now days, it is generally recognized by the educational society as well as industry that the assistance among the vehicles as well as road transportation devices which certainly enhance driver's security along with road performance and lowers the atmospheric impact. Considering this specifically, the growth of VANETS has got more interest as well as study efforts. Significantly more efforts have been performed to use a typical program to accomplish inter-vehicle communications (IVCs) [1]. VANETS have risen beyond the requirements to offer the developing variety of cellular items which can be very easily employed in vehicles [11]. In VANET innovation of $\mathrm{Wi}-\mathrm{Fi}$, Bluetooth as well as various other mobile connectivity methods is used since building conventional for relationship involving nodes for instance car or truck to be able to car or truck along with car or truck to be able to path area model. This multilevel increase the risk for architecture with VANET for the reason that any node is usually vehicle that could talk with various other node and also additionally made use of web center by employing street area units. Therefore features with VANET including great mobility, street topology and also absolutely no limits with multilevel size enabling it distinct from various other ad-hoc multilevel including MANET as well as also make challenging environment to develop efficient networks [12]. V2V communications are usually based on the IEEE 802.11p [7]. Thus the immediate connection among cars or trucks using an Advertising Hoc circle, often known as inter-vehicle Communication (IVC) as well as auto advertising hoc cpa affiliate networks (VANETS), is actually a revolutionary approach. Around Evaluation for you to a wireless program, IVC possess various important aspects: reduced latency due to immediate connection, larger protection and without support charger [2]. The particular qualities associated with VANETS enable the introduction of desirable innovative services.

\section{ADHOC ON DEMAND DISTANCE VECTOR-RELIABILITY (AODV-R)}

Inside AODV-R routing protocol, $\mathrm{R}$ is pertaining to reliability. AODV is commonly a reactive routing protocol, any time i.e. the item makes a path among the cause node and also the receiver node simply just in need. AODV bring either uni-cast as well as multicast routing. Every time a multilevel node requires a hyperlink, this transports some sort of the navigation inquire (RREQ) sales message for the nearby vehicles. [23]. Pretty much every node keeps this specific RREQ help keep this node the idea listened to via in addition to toward other sorts of nodes. This system associated together with creating the prior hop is known as backward mastering. If one of many intermediate nodes carries a path for the location, it reacts time for the source node with this path. If in case several reactions obtain in the origin node, subsequently the item utilizes the path using the small number of hops.

\section{CLUSTERING AND ANT COLONY OPTIMIZATION}

\subsection{Clustering (Data Aggregation)}

The use of clustering in proposed technique allows VANETS nodes to refuse data in certain area. Thus instead of every board unit will communicate with its data and RSU, it will simply sends its data to nearest authentication on board unit thus lesser transmission energy is going to be disputed as well as lesser chance of link failures will be there. Thus it automatically leads to less end to end delay because the communication distance is less in proposed AODV-R protocol.

\subsection{Ant Colony Optimization}

Ant Colony Optimization is particularly a routing technique especially helpful to find out shortest path between two nodes. For that purpose the entry to speediest distance algorithm lets practices so that you can find that route among the sender and also device containing much less yardage Hence the path chosen by simply bare minimum yardage algorithm comes up with project AODV-R. While AODV-R uses ACO algorithm i.e. it can be improved using ant-colony optimization for selecting shortest path algorithm. The ACO algorithm aims to select the best route including the route reliability, packet delivery ratio, control overhead, end to end delay, link failures.

\section{RELATED WORK}

Lars, Wischhof et al. [1] represented a skill intended for scalable information and facts dissemination within highly cellular adhoc communities, the idea states process concentrated info abstraction plus dissemination (SODAD) together with this approach one particular application can be 
shown i.e. self-organizing traffic-information system (SOTIS). Saleh, Yousefi et al. [2] introduced the comprehensive study connected with challenges throughout with these networks that targets the different issues as well as solutions. It has characteristics which might be different coming from generic MANETS. Tarik, Taleb et al. [3] suggested the usage of details on vehicles movement information to know a possible link breakage. The scheme used behind is to send only particular and well known packets called as best packets. Zhan, Huaweri et al. [4] it proposes two known algorithms i.e. Adhoc on-demand distance vector protocol (AODV) and optimized link state routing protocol (OLSR) are analyzed and compared. Yue, Lui et al. [5] focused on vehicular networks which contain wide range of services, information and entertainment applications. It provides a revision for the researchers related to vehicular adhoc network. Sandhaya, Kohli et al. [6] introduced the advantages and disadvantages of the routing protocols. Ray, Hunt et al. [11] presented a review of wireless access standards for VANETS through this access their benefits and limitations. Yatendra, Mohan et al. [12] focused on the study of various the navigation methodologies and also continuous investigation inside VANET using their worth \& mistakes, that can be used even more development with pre-existing project or maybe growth of brand new useful and much more reputable methodologies with regard to many of the programs inside VANET. Kazemi, Babak et al. [18] proposed approach for routing in VANETS used for highways, i.e. ACO algorithm. The end result displays how the suggested system considerably outperforms equivalent methods inside literature. Eiza, Mahmoud Hashem et al. [21] represented a new vehicular dependability design to offer the particular reputable course-plotting inside VANETs. This offers the particular identified project i.e. adhoc on-demand yardage vector (AODV) course-plotting project to obtain powerful courseplotting more that offers AODV so that you can AODVR. Manchanda, Puneet et al. [23] this paper proposes an algorithm that includes the reliability as well as delay factor in the existing AODV-R protocol to decrease the end to end delay. The protocol also reduced the routing overhead. Macedo, Ricardo et al. [27] the work shows a great trial and error overall performance study comparing between singlepath as well as multipath direction-finding solutions, hoping to recognize their own habits so that you can support the perception of effective direction-finding protocols. Li Gen, Maode et al. [28] propose the Fuzzy Multiple Attribute Decision Routing (FMADR) scheme by which we characterize the candidate vehicles with multiple attributes and select the candidates for next hop transmission by the multiple attribute decision making (MADM) approach. Kristiana, Lisa et al. [34] provides a brief overview of three dimensional challenges and evaluates existing forwarding method where propagation and position-based routing are considered, which influences the design. The proposed forwarding method, termed Vehicle to-Vehicle Urban Network (V2VUNet) filters out neighbor nodes with potential loss transmission

\section{METHODOLOGY}

1. First of all network deployment is done i.e. road side units, vehicular nodes etc in a given area.

2. Some nodes will be initiated act as senders. Each sender demands a path between certain predefined locations.

3. Now each road side unit aggregates the demand of different nodes and apply time division multiple access to find the accepted routes.
4. Now ant colony optimization comes in action to find shortest path between each sender and its receiver using pheromone trails.

\subsection{Methodology}

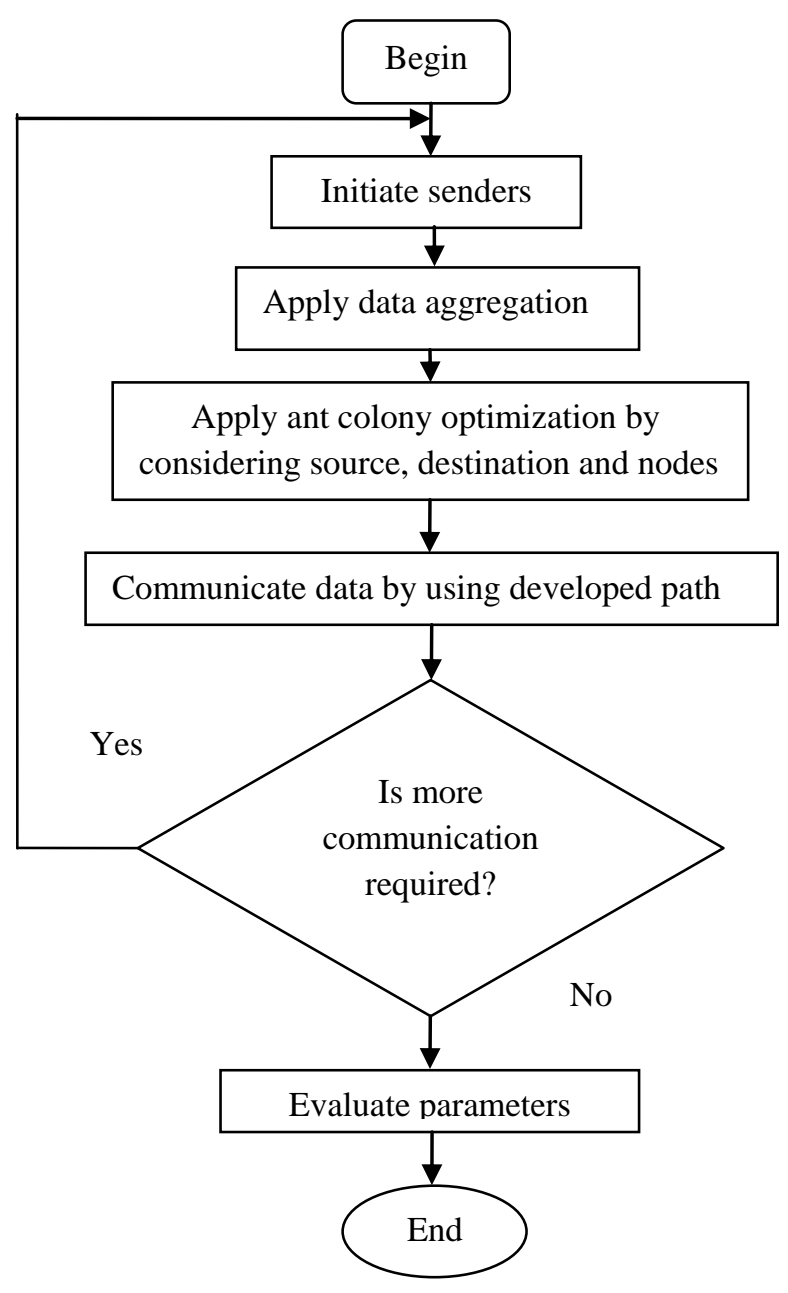

Fig1: Flowchart of the Proposed Technique [21]

\section{RESULTS}

For experimentation and implementation the proposed technique is evaluated using MATLAB tool u2013a. The evaluation of proposed technique is done on the basis of following parameters i.e. packet delivery ratio, control overhead ratio, end-to-end delay and link failures based on the velocity with data packet size 1000 and no of nodes 10 .

Table no. 1 Result Analysis of Different parameters

\begin{tabular}{|c|l|l|l|l|}
\hline $\begin{array}{c}\text { Velocity } \\
\text { in third } \\
\text { lane }\end{array}$ & $\begin{array}{l}\text { Packet } \\
\text { delivery } \\
\text { ratio }\end{array}$ & $\begin{array}{l}\text { Control } \\
\text { overhead } \\
\text { ratio }\end{array}$ & $\begin{array}{l}\text { End to } \\
\text { end } \\
\text { delay }\end{array}$ & $\begin{array}{l}\text { Link } \\
\text { failures }\end{array}$ \\
\hline 60 & 70.3025 & 28.6328 & 0.0072 & 75 \\
\hline 80 & 71.5149 & 32.8814 & 0.0067 & 67 \\
\hline 100 & 69.0906 & 24.3816 & 0.0014 & 65 \\
\hline 120 & 70.3027 & 28.6746 & 0.0011 & 72 \\
\hline 140 & 67.8785 & 20.1421 & 0.0050 & 67 \\
\hline
\end{tabular}




\subsection{Packet delivery ratio}

It shows the normal ratio of the volume of effectively acquired data packets at the receiver node to the volume of data packets delivered.

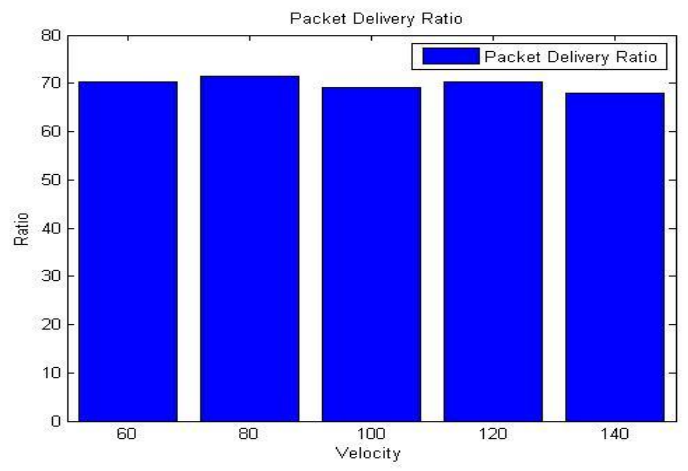

Fig2: Packet delivery ratio $(\%)$

The above fig. 2 represents the evaluation of new clustering ant Colony Optimization based routing protocol AODV-R shows packet delivery ratio reduces when velocity increases. Where $\mathrm{x}$-axis represents the velocity and $\mathrm{y}$-axis represents the Packet delivery ratio.

\subsection{Control overhead ratio}

It defines the ratio of the total amount of routing request messages generated to the total amount of data messages delivered.

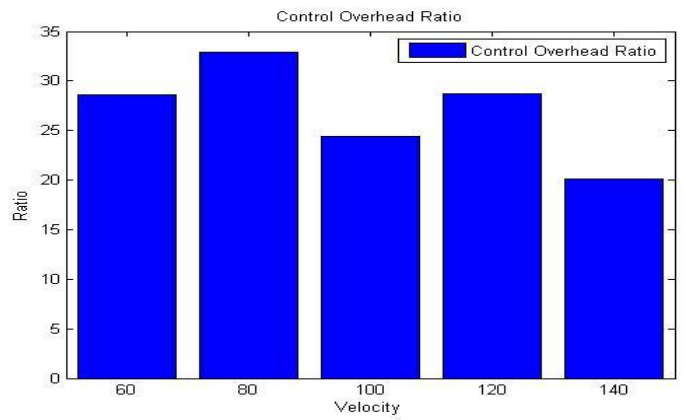

Fig3: Control overhead ( time in seconds)

The above fig. 3 represents the evaluation of new clustering ant Colony Optimization based routing protocol AODV-R shows the control overhead ratio increases when velocity increases. Where $\mathrm{x}$-axis represents the velocity and $\mathrm{y}$-axis represents the control overhead ratio.

\subsection{End -to-end delay}

End-to-End delay generally describes the time among the sender and destination the data packets which are effectively received at the destination node.

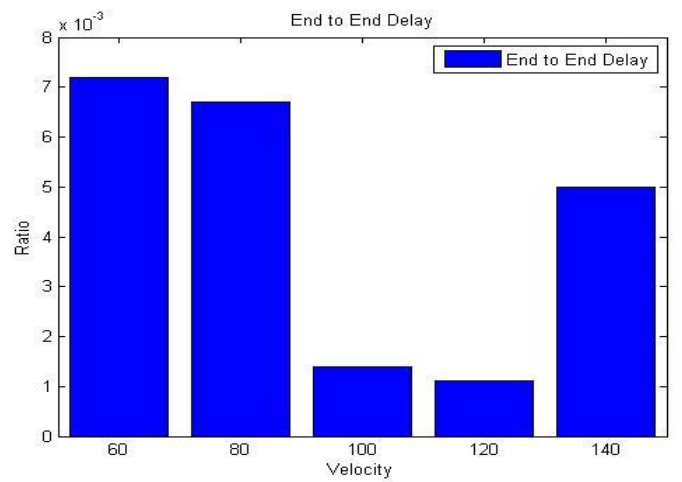

Fig 4: End to end delay (time in seconds)

The above fig.4 represents the evaluation of new clustering ant Colony Optimization based routing protocol AODV-R shows end to end delay increases when velocity increases. Where $\mathrm{x}$-axis represents the velocity and $\mathrm{y}$-axis represents the Delay (in sec).

\subsection{Link Failures}

It represents the number of link breakages that take place during the data transmission

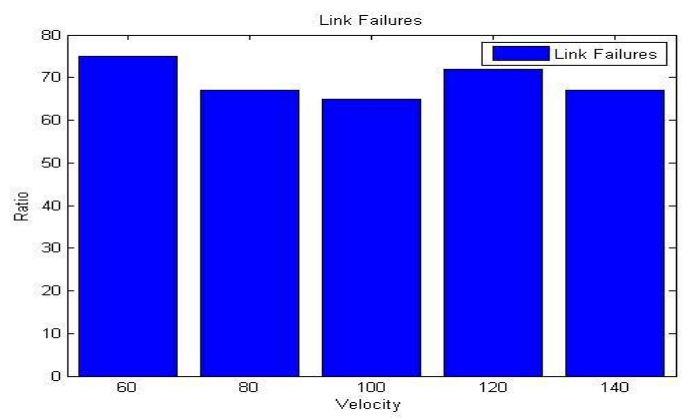

Fig 5: Link Failures

The above fig. 5 represents the evaluation of new clustering ant Colony Optimization based routing protocol AODV-R shows link failures increases when velocity increases. Where $\mathrm{x}$-axis represents the velocity and $\mathrm{y}$-axis represents the link failures.

\section{CONCLUSION AND FUTURE WORK}

In VANETs the communication links are at extreme risk of disconnection. There is a need for an effective routing in algorithm that can deal with the high mobility of vehicles as well as provide better data aggregation and route selection. In this paper we have implemented AODV-R by using new clustering ant Colony Optimization and the results of the same have shown significant improvement. The evaluation for new clustering ant Colony Optimization based AODV-R is done using parameters like packet delivery ratio, control overhead ratio, end-to-end delay and link failures. Evaluated AODV-R selects the most reliable path which helps in reducing the possibility of link breakages as well as responds better tochanges in network topology.

\section{REFERENCES}

[1] L. Wischhof, A. Ebner and H. Rohling, "Information Dissemination inSelf-Organizing Intervehicle Networks",IEEE Transaction on Intelligent Transportation Systems, Vol. 6, No. 1, March 2005.

[2] Yousefi, Saleh, Mahmoud Siadat Mousavi, and Mahmood Fathy. "Vehicular ad hoc networks 
(VANETs): challenges and perspectives." In ITS Telecommunications Proceedings, 2006 6th International Conference on, pp. 761-766. IEEE, 2006.

[3] Taleb, Tarik, Ehssan Sakhaee, Abbas Jamalipour, Kazuo Hashimoto, Nei Kato, and Yoshiaki Nemoto. "A stable routing protocol to support ITS services in VANET networks." Vehicular Technology, IEEE Transactions on 56, no. 6 (2007): 3337-3347.

[4] Huawei, Zhan, and Zhou Yun. "Comparison and analysis AODV and OLSR routing protocols in ad hoc network." In Wireless Communications, Networking and Mobile Computing, 2008. WiCOM'08. 4th International Conference on, pp. 1-4. IEEE, 2008.

[5] Liu, Yue, Jun Bi, and Ju Yang. "Research on vehicular ad hoc networks." InControl and Decision Conference, 2009. CCDC'09. Chinese, pp. 4430-4435. IEEE, 2009.

[6] Kohli, Sandhaya, Bandanjot Kaur, and Sabina Bindra. "A comparative study of Routing Protocols in VANET." Proceedings of ISCET (2010).

[7] Rondinone, Michele, and Javier Gozalvez. "Exploiting multi-hop connectivity for dynamic routing in VANETs." In Wireless Communication Systems (ISWCS), 2011 8th International Symposium on, pp. 111-115. IEEE, 2011.

[8] Venkata, M. D., MM Manohara Pai, Radhika M. Pai, and Joseph Mouzna. "Traffic monitoring and routing in VANETs - A cluster based approach." In ITS Telecommunications (ITST), 2011 11th International Conference on, pp. 27-32. IEEE, 2011.

[9] Song, Chao, Ming Liu, Yonggang Wen, Jiannong Cao, and Guihai Chen. "Buffer and switch: An efficient roadto-road routing scheme for vanets." In Mobile Ad-hoc and Sensor Networks (MSN), 2011 Seventh International Conference on, pp. 310-317. IEEE, 2011.

[10] Kakarla, Jagadeesh, S. Siva Sathya, and B. Govinda Laxmi. "A Survey on Routing Protocols and its Issues in VANET." (2011).

[11] Zeadally, Sherali, Ray Hunt, Yuh-Shyan Chen, Angela Irwin, and Aamir Hassan. "Vehicular ad hoc networks (VANETS):status,results,andchallenges." Telecommunic ation Systems 50, no. 4 (2012): 217-241.

[12] Sharma, Yatendra Mohan, and Dr Saurabh Mukherjee. s"A Contemporary Proportional Exploration of Numerous Routing Protocol in VANET."International Journal of Computer Applications (0975-8887) Volume (2012).

[13] Hou, Jie, Lei Han, Jiqiang Liu, and Jia Zhao. "Secure and efficient protocol for position-based routing in VANETs." In Intelligent Control, Automatic Detection and High-End Equipment (ICADE), 2012 IEEE International Conference on, pp. 142-148. IEEE, 2012.

[14] Wu, Celimuge, Satoshi Ohzahata, and Toshihiko Kato. "Routing in vanets: A fuzzy constraint q-learning approach." In Global Communications Conference (GLOBECOM), 2012 IEEE, pp. 195-200. IEEE, 2012.

[15] Ghafoor, Huma, N. D. Gohar, and Rizwan Bulbul. "Anchor-based Connectivity Aware Routing in VANETs." In Wireless Communications, Networking and Mobile Computing (WiCOM), 2012 8th International Conference on, pp. 1-6. IEEE, 2012
[16] Wu, Celimuge, Satoshi Ohzahata, and Toshihiko Kato. "A loss-tolerant scheme for unicast routing in VANETs using network coding." In Wireless Communications and Mobile Computing Conference (IWCMC), 2013 9th International, pp. 1058-1063. IEEE, 2013.

[17] Wu, Celimuge, Satoshi Ohzahata, and Toshihiko Kato. "Can we generate efficient routes by using only beacons? Backbone routing in VANETs." In Personal Indoor and Mobile Radio Communications (PIMRC), 2013 IEEE 24th International Symposium on, pp. 2929-2934. IEEE, 2013.

[18] Kazemi, Babak, Masoumeh Ahmadi, and Siamak Talebi. "Optimum and reliable routing in VANETs: An opposition sbased ant colony algorithm scheme." In Connected Vehicles and Expo (ICCVE), 2013 International Conference on, pp. 926-930. IEEE, 2013.

[19] Kabir, Md Humayun. "Research Issues on Vehicular Ad hoc Network."International Journal of Engineering Trends and Technology (IJETT)-Volume 6.2013.

[20] Karande, Mr Nikhil D., and Ms Kushal K. Kulkarni. "Efficient routing protocols for vehicular adhoc network." In International Journal of Engineering Research and Technology, vol. 2, no. 1 (January-2013). ESRSA Publications, 2013.

[21] Eiza, Mahmoud Hashem, Qiang Ni, Thomas Owens, and Geyong Min. "Investigation of routing reliability of vehicular ad hoc networks." EURASIP journal on wireless communications and networking 2013, no. 1 (2013): $1-15$

[22] Wu, Celimuge, Satoshi Ohzahata, and Toshihiko Kato. "Flexible, Portable, and Practicable Solution for Routing in VANETs: A Fuzzy Constraint Q-Learning Approach." Vehicular Technology, IEEE Transactions on 62, no. 9 (2013): 4251-4263.

[23] Manchanda Puneet and Bangar Parvinder."Modified AODV-R Routing Protocol.” International Journal of Engineering, Applied and Management Sciences Paradigms, Vol. 16,(2014) 2320-6608

[24] Chitra, M., S. Sivasathya, and B. Muthamizh "Performance Analysis of Topology based Routing Protocols in VANET." (2014)

[25] Zhang, Xiaomei, Xiaolei Dong, Naixue Xiong, Jie Wu, and Xiuqi Li. "Fault-aware flow control and multi-path routing in VANETs." Peer-to-Peer Networking and Applications (2014): 1-18

[26] Gupta, Divya, and Rajesh Kumar. "An improved genetic based Routing Protocol for VANETs." In Confluence The Next Generation Information Technology Summit (Confluence), 2014 5th International Conference-, pp. 347-353. IEEE, 2014.

[27] Macedo, Ricardo, Robson Melo, Aldri Santos, and Michele Nogueira. "Experimental performance comparison of single-path and multipath routing in VANETs." In Global Information Infrastructure and Networking Symposium (GIIS), 2014, pp. 1-6. IEEE, 2014.

[28] Li, Gen, Maode Ma, Chunfeng Liu, and Yantai Shu. "Fuzzy Multiple Attribute Decision Routing in VANETs." In Communication Systems (ICCS), 2014 IEEE International Conference on, pp. 564-568. IEEE, 2014. 
[29] Dua, Amit, Neeraj Kumar, and Seema Bawa. "A systematic review on routing protocols for vehicular ad hoc networks." Vehicular Communications 1, no. 1 (2014): 33-52.

[30] Sharef, Baraa T., Raed A. Alsaqour, and Mahamod Ismail. "Vehicular communication ad hoc routing protocols: A survey." Journal of network and computer applications 40 (2014): 363-396.

[31] Kaiwartya, Omprakash, and Sudhakar Kumar. "Geocast routing: Recent advances and future challenges in vehicular adhoc networks." In Signal Processing and Integrated Networks (SPIN), 2014 International Conference on, pp. 291-296. IEEE, 2014.
[32] Khairnar, Vaishali D., and Dr SN Pradhan. "Simulation Based: Study and Analysis of Routing Protocol inVehicular Ad-hocNetwork environment." arXivpreprintarXiv:1403.6013 (2014).

[33] He, Yang, Wenjun Xu, and Xuehong Lin. "A Stable Routing Protocol for Highway Mobility over Vehicular Ad-Hoc Networks." In Vehicular Technology Conference (VTC Spring), 2015 IEEE 81st, pp. 1-5. IEEE, 2015.

[34] Kristiana, Lisa, Corinna Schmitt, and Burkhard Stiller. "Investigating a Reliable Inter-vehicle Network in a Three Dimensional Environment." Ulmer InformatikBerichte (2015): 16. 